\title{
Attitude Towards COVID-19 Vaccination Among Healthcare Workers: A Systematic Review
}

\author{
Mohammedamin Hajure (D) \\ Mandaras Tariku (D) ${ }^{2}$ \\ Firomsa Bekele $\mathbb{D}^{3}$ \\ Zakir Abdu (D) ${ }^{\prime}$ \\ Aman Dule (D) ${ }^{\prime}$ \\ Mustefa Mohammedhussein (ID) \\ Tesfaye Tsegaye ${ }^{3}$ \\ 'Department of Psychiatry, College of \\ Health Science, Mettu University, Mettu, \\ Ethiopia; ${ }^{2}$ Department of Psychiatry, \\ College of Health and Medical Science, \\ Haramaya University, Harar, Ethiopia; \\ ${ }^{3}$ Department of Pharmacy, College of \\ Health Science, Mettu University, Mettu, \\ Ethiopia
}

Correspondence: Mohammedamin Hajure Department of Psychiatry, College of Health Sciences, Mettu University, PO Box: 38I,

Mettu, Ethiopia

Tel +25I-913596045

Email sikoado340@gmail.com
Introduction: Availability and accessibility of a safe COVID-19 vaccine do not necessarily guarantee an effective means to mitigate the pandemic. However, the fragile hero's or health care worker's attitude toward the vaccine is of paramount importance to promote its acceptance. So, the current review aims to provide the latest assessment of healthcare workers' attitudes toward the COVID-19 vaccination and its contributing factor worldwide.

Methods: Peer-reviewed surveys in English indexed via an electronic database in Google Scholar, Science Direct and PubMed were systematically searched. The review was carried out per the Preferred Reporting Items for Systematic Reviews and Meta-analyses (PRISMA2009) and registered on PROSPERO (CRD42021265534).

Results: Originally 8039 articles were searched from three databases PubMed, Science direct, and Google scholar. Finally, 24 studies met the inclusion criteria and made the root for the estimates of the attitude of COVID -19 vaccinations. In about two-thirds of the studies, respondents showed a positive attitude ( $\geq 50 \%)$ toward COVID-19 vaccination. However, in about one-quarter of the studies, a negative attitude $(<50 \%)$ against vaccination was reported. Factors related to the attitude of healthcare workers toward COVID-19 vaccination include age, sex, profession, concerns about the safety of vaccines and fear of COVID-19, trust in the accuracy of the measures taken by the government, flu vaccination during the previous season, comorbid chronic illness, history of recommendation, and depression symptoms in the past week.

Conclusion: Although most studies report that healthcare workers have a positive attitude toward COVID-19 vaccination, quite a few surveys mention negative attitudes towards the use of vaccines, which may reflect missed opportunities or challenges for the international efforts aimed at mitigating the pandemic. Still, we need to continue to make more efforts to change the attitudes of the uncertain healthcare workers to increase the uptake of the vaccine and deal with the multi-faceted impact of infection.

Keywords: attitude, SARS-COV-2, vaccination, healthcare workers

\section{Introduction}

The COVID-19 pandemic has caused a huge number of deaths. Globally, as of 5 July 2021, there have been 183,198,019 confirmed cases of COVID-19, including $3,971,687$ deaths reported to WHO. ${ }^{1}$ As of 8 July 2021, a total of 3,032,217,959 vaccine doses have been administered and are likely to continue to have significant impacts on healthcare communication. ${ }^{2}$

A WHO report showed that nearly all COVID deaths in the US are now of people who were not vaccinated, a staggering demonstration of how effective the shot has been. ${ }^{3}$ Following the announcement of the first emergency use authorization for COVID-19 vaccine in December 2020 by the Food and Drug 
Administration, ${ }^{4}$ priority to get the vaccine was given for risky groups of populations such as healthcare workers and medical students. While immunization has effectively decreased the morbidity and mortality of infectious disease worldwide, a far-reaching trust of vaccines among general populations can be impaired by different factors contributing to the low uptake of the vaccine. These include media demonstrating less trust in the vaccine, ${ }^{5}$ a low opportunity of getting the vaccination, perceived risk of infection, economic problems, ${ }^{6-8}$ presence of depression symptoms, fear of passing on the disease to family, ${ }^{9}$ and knowledge and attitude of healthcare workers toward vaccination. ${ }^{10}$

Taking into account the types of vaccine, the study shows that the controversy about the severity of common side effects of AstraZeneca's COVID-19 vaccine is related to the uncertainty of healthcare workers about the COVID19 vaccine. ${ }^{11}$ In other respects, in terms of vaccine effectiveness, the study showed that between 77.0 and $90.7 \%$ of screened physicians in Colombia would accept vaccination against COVID-19 when the vaccine efficacy was 60 and $80 \%$, respectively, at the time when Sinovac (Beijing, China) (Coronavac) and Pfizer-BioNTech (BNT162b2) vaccines types have reported $50 \%$ and $95 \%$ efficacy. ${ }^{12}$

In the context of the current worldwide health and financial crisis from COVID-19, vaccines have become the primary anticipated means to end its effects. ${ }^{13}$ So reducing the spread of new COVID-19 infection is anticipated through vaccination of all people, with particular concern for a risky population like healthcare workers. ${ }^{14}$ Besides the efficient community-level health measures like wearing face masks, social distancing, avoidance of populated areas, awareness creation, and handwashing, effective vaccination is vital to prevent morbidity and mortality of the pandemic. ${ }^{15-17}$

In addition to the complex process of vaccine development and experimentation which was taken for not less than one and half years, ${ }^{18}$ dissemination and acceptance among the general population have been further headaches, ${ }^{13,19}$ with an understanding of attaining herd immunity by infection. Healthcare workers' recommendations are vital to increasing the uptake of the vaccine among the general population via the promotion of health communication and modeling. ${ }^{20-22}$ Results of a community-based study involving Italian undergraduate students reported a good level of knowledge and acceptance of COVID-19 vaccination, revealing a sufficient flow of information or an efficient communication campaign among this population. $^{23}$ This supports an imperative role of the media campaign and public health communications in combating the pandemic via behavioural change ${ }^{24}$ and targeting a barrier to vaccine uptake. ${ }^{25}$

The previous study was confined to a single or a few countries. So better to make a more common understanding of the attitude of healthcare workers internationally and to identify common determinants to help design better measures to improve COVID-19 vaccination uptake as the COVID-19 vaccination worries require collaborative effort planners, policymakers, and the community at large.

In addition, earlier studies have shown that vaccination of healthcare workers with influenza vaccine decreases patient mortality and staff absenteeism. ${ }^{26}$ It would be reasonable to expect a similar benefit with the COVID19 vaccination. Taking this into consideration, it is generally assumed that creating an intellectual understanding of the use of COVID-19 vaccine and budding factors of healthcare workers behaviour is crucial for designing sustainable health communications to instigate the acceptance and fruitful intervention of COVID-19 vaccination.

Thus, we conducted a systematic review of qualitative literature to make available wide-ranging evidence/consensus on the attitude of healthcare workers and its correlates towards COVID-19 vaccination intending to identify barriers to and create awareness of public health strategists, researchers and policymakers take part in planning the implementation of vaccine to widespread vaccination.

\section{Methods and Materials}

\section{Search Strategy}

This study attempted to summarize the results of earlier published studies on the attitude towards SARS-CoV-2 vaccination among healthcare workers. The study was carried out as per the guideline of PRISMA-2009 from three databases: PubMed $(n=211)$, Science direct $(n=65)$, and Google scholar $(\mathrm{n}=7450){ }^{27}$ The seven authors, named MH, MT, FB, ZA, AD, MM, and TT, conducted the review. Initially, $\mathrm{MH}, \mathrm{MT}$, and $\mathrm{FB}$ undertook screening of the titles and abstracts of the articles for exclusion blindly via consistent check for any differences observed. $\mathrm{ZA}$ and $\mathrm{AD}$ did the screening of full texts for the eligibility, and TT and MM checked for the compatibility of the screened articles as per the stated criteria. There was a good level of agreement between the authors. Mendeley Desktop version 1.19.8 was used to remove redundant files and further research was also searched using forward citation tracking (Google Scholar). 
Authors conducted search for peer-reviewed original research works using electronic databases (PubMed, Science direct and Google Scholar). Mesh terms used for the entire database were as follows: (( ("attitude"[MeSH Terms] OR "attitude"[All Fields]) AND towards[All Fields] AND ("sars-cov-2"[MeSH Terms] OR "sars-cov2"[All Fields] OR "sars cov 2"[All Fields])) AND ("vaccination"[MeSH Terms] OR "vaccination"[All Fields])) AND ("health personnel"[MeSH Terms] OR ("health"[All Fields] AND "personnel"[All Fields]) OR "health personnel"[All Fields] OR ("healthcare"[All Fields] AND "workers"[All Fields]) OR "healthcare workers"[All Fields]). Studies published during the COVID-19 pandemic and fulfilling the inclusion were searched for from May to 20 July 2021.

\section{Eligibility Criteria}

We considered studies eligible for review if they were published in the English language, available in full texts, and included outcome under study. Studies lacking original data like short communications, commentary, metaanalysis, preprints, and original articles with inaccessible full texts are excluded. The review was registered on PROSPERO (registration number: CRD42021265534). The attitude of healthcare workers was classified as positive $(\geq 50 \%)$ and negative $(<50 \%)$, which was the magnitude of acceptance/willingness or refusal, hesitancy, or reluctance reported among respondents.

\section{Data Abstraction}

Data were extracted by two authors autonomously and the rest of the authors confirmed the process. The details of extraction include the names of the first authors, year of study, country, sample size and design, socio-demographic data (age of respondents, gender, and types of profession), the outcome of interest, and their correlates. A search for the main outcome was the attitude towards COVID-19 vaccination among healthcare workers based on the following question: "Do you intend to take the vaccine if the option is available to you?" "Yes, or No" is different as stated in the majority of the survey (See Supplemental Information). The results were reported narratively and also presented descriptively in the form of tables and figures.

\section{Methodology Quality Assessment}

A National Institute of Health quality assessment tool for observational cohort and cross-sectional studies was used to delineate the quality of methodology. Accordingly, from the tool criteria numbers $1-6,11,14$ were used to rate the quality of the included article. As per the tool, all queries were filled with "yes", "no" or "cannot determine" and "not applicable" and "not reported". ${ }^{23}$ Accordingly, 15 articles were good, ${ }^{13,24-37} 6$ were fair, ${ }^{31,38-42}$ and 3 were poor. $^{47-49}$

\section{Results}

\section{Search Results}

The initial literature search yielded a total of 8039 articles through database searches such as google scholar, ScienceDirect, PubMed, and other sources. Of these, 4230 were excluded due to duplication, thus 3809 were left remaining. Then, after screenings of their title and abstract, 3720 were excluded, resulting in 89 articles becoming candidates for full-text screening. Lastly, 24 samples have become potentially eligible to undertake the systematic review (Figure 1).

\section{Overview of Studies Included in This Review}

A total of 24 published articles were included in the current review. Included studies were conducted in the USA $(n=2)$, Saudi Arabia $(n=2)$, Ghana $(n=2)$, Italy $(n=2)$, France, Greece, Congo, Poland, Romania, Taiwan, Canada, Colombia, Slovenia, the United Arab Emirates, Arabic- speaking countries, Pakistan, Nepal, Belgium, Egypt, and Israel (Table 1).

All of the studies used cross-sectional surveys and about two-thirds of the studies $(\mathrm{n}=16)$ used online surveys or interviews. Apart from two studies, France ${ }^{41}$ and Congo $^{33}$ almost all others $(\mathrm{n}=22)$ were published in the year 2021.

The sample size of included studies ranged from 140 to 5287 (average sample size $=613$ ). In most of the samples, the age of respondents lies between 25-40 years. Physicians dominate among healthcare workers in most of the studies $(n=13)$, typically from France, ${ }^{41}$ Egypt,${ }^{47}$ Ghana, ${ }^{48}$ Congo, ${ }^{33}$ Greece, ${ }^{45}$ Italy, ${ }^{44}$ Poland, ${ }^{32}$ Saudi Arabia, ${ }^{36}$ Romania, ${ }^{29}$ Colombia, ${ }^{12}$ Slovenia $^{34}$ and the United Arab Emirates. ${ }^{42}$ However, nurses dominate among studies conducted in $\mathrm{Nepal}^{46}{ }^{46}$ Belgium, ${ }^{35}$ the USA, ${ }^{40}$ Canada, ${ }^{38}$ and Saudi Arabia. ${ }^{39}$ Most of the participants were females in the majority of the samples $(n=16)$, while males predominate in samples of Israel, ${ }^{43}$ Saudi Arabia, ${ }^{36}$ Arabic-speaking countries, ${ }^{37}$ and 


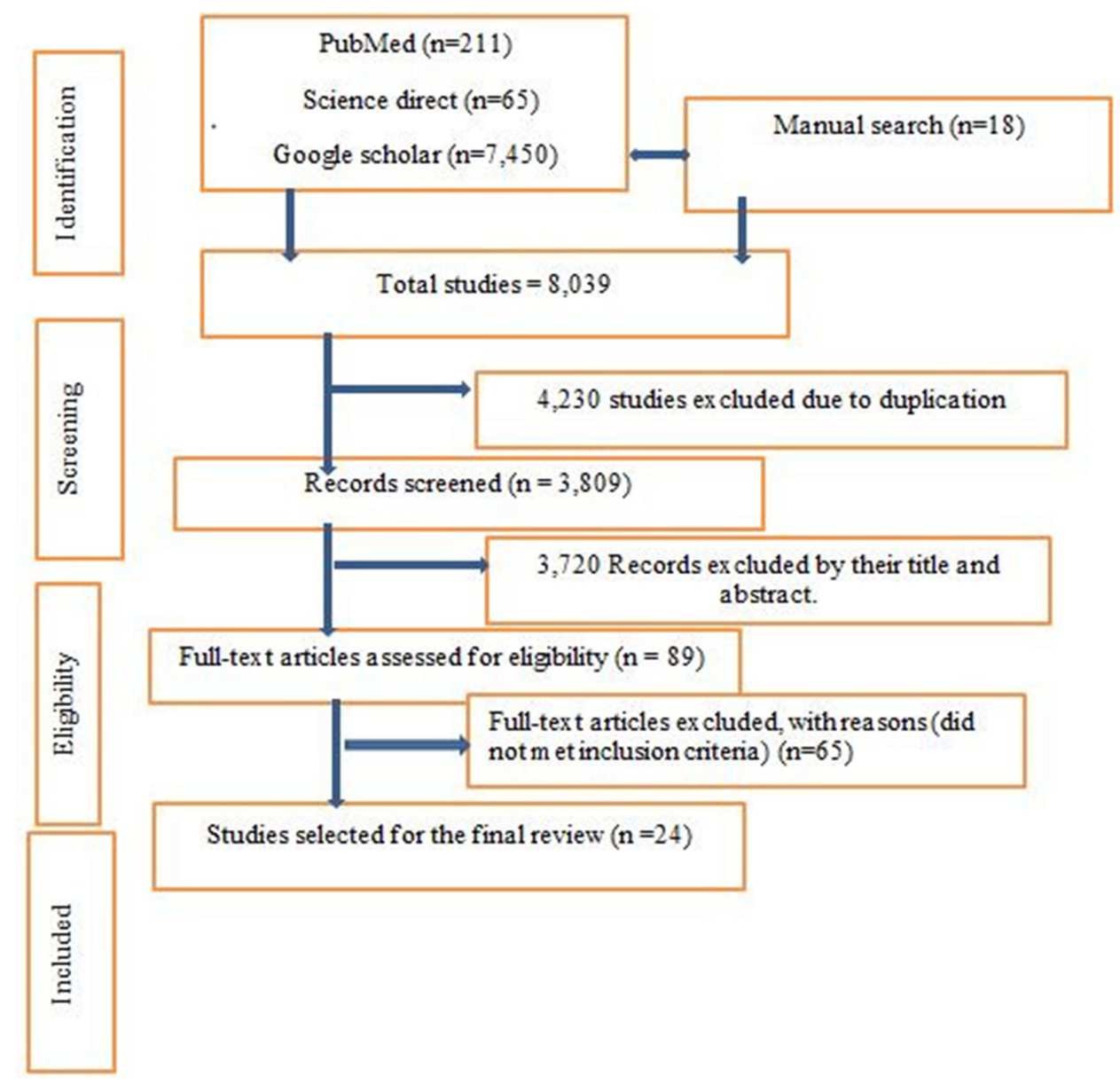

Figure I PRISMA flow diagram of the study.

Note: Adapted from Page MJ, McKenzie JE, Bossuyt PM, Boutron I, Hoffmann TC, Mulrow CD, et al. The PRISMA 2020 statement: an updated guideline for reporting systematic reviews. BMJ. 2021;372:n7I. doi: 10.1I36/bmj.n7I. ${ }^{62}$

Poland. $^{32}$ There are approximately equal ratio of participation among both sexes in Ghana, ${ }^{48}$ Congo, $^{33}$ and Greece ${ }^{45}$ (Table 1).

\section{Attitude Toward COVID-19 Vaccination}

Studies conducted among different countries or subgroups of healthcare professionals to make a comprehensive assessment of vaccination uptake showed significant variation. The attitude of healthcare professionals was explained in terms of a general anti-vaccine stand including hesitancy, refusal, acceptance/non-acceptance, or unwillingness/willingness to take COVID-19 vaccine or recommendation to others.

This review showed that in about two-thirds of the studies, respondents showed a positive attitude $(\geq 50 \%)$ toward COVID-19 vaccination, while in about one-quarter of the studies negative attitude $(<50 \%)$ against vaccination was reported. The lower and higher rate of attitude toward COVID-19 uptake was reported in Egypt and United Arab
Emirates respectively. The review indicated that a more negative attitude was observed in the survey among African and Asian studies (Table 2).

In the current review stratified by country, there are lower anti-vaccination approaches or more positive attitudes $(\geq 50 \%)$ toward COVID-19 vaccine in Colombia $(90.7 \%){ }^{12}$ Poland $(82.95 \%){ }^{32}$ and the United Arab Emirates $(85 \%){ }^{42}$ In contrast to this, higher anti-vaccination or negative attitudes were reported in Arabic-speaking countries (26.7\%), ${ }^{37}$ Nepal (38.3\%), ${ }^{46}$ Taiwan $(23.4 \%),{ }^{31}$ Congo (27.7\%), ${ }^{33}$ Israel, $^{43}$ and Belgium (37.1\%), ${ }^{35}$ with different degrees of certainty. Healthcare workers in most of the internationally reported samples, Italy $(67 \%$ to $75 \%),{ }^{30,44}$ Saudi Arabia (50.5\% to $\left.51.3 \%\right),{ }^{36,39}$ the USA $(57.5 \%),{ }^{15}$ Romania (69\%), ${ }^{29}$ Ghana $(67 \%),{ }^{48}$ Slovenia $(59 \%),{ }^{34}$ France $(76.9 \%),{ }^{41}$ and Greece $(78.5 \%),{ }^{45}$ showed a moderate approach or attitude toward COVID-19 vaccine use or uptake. 


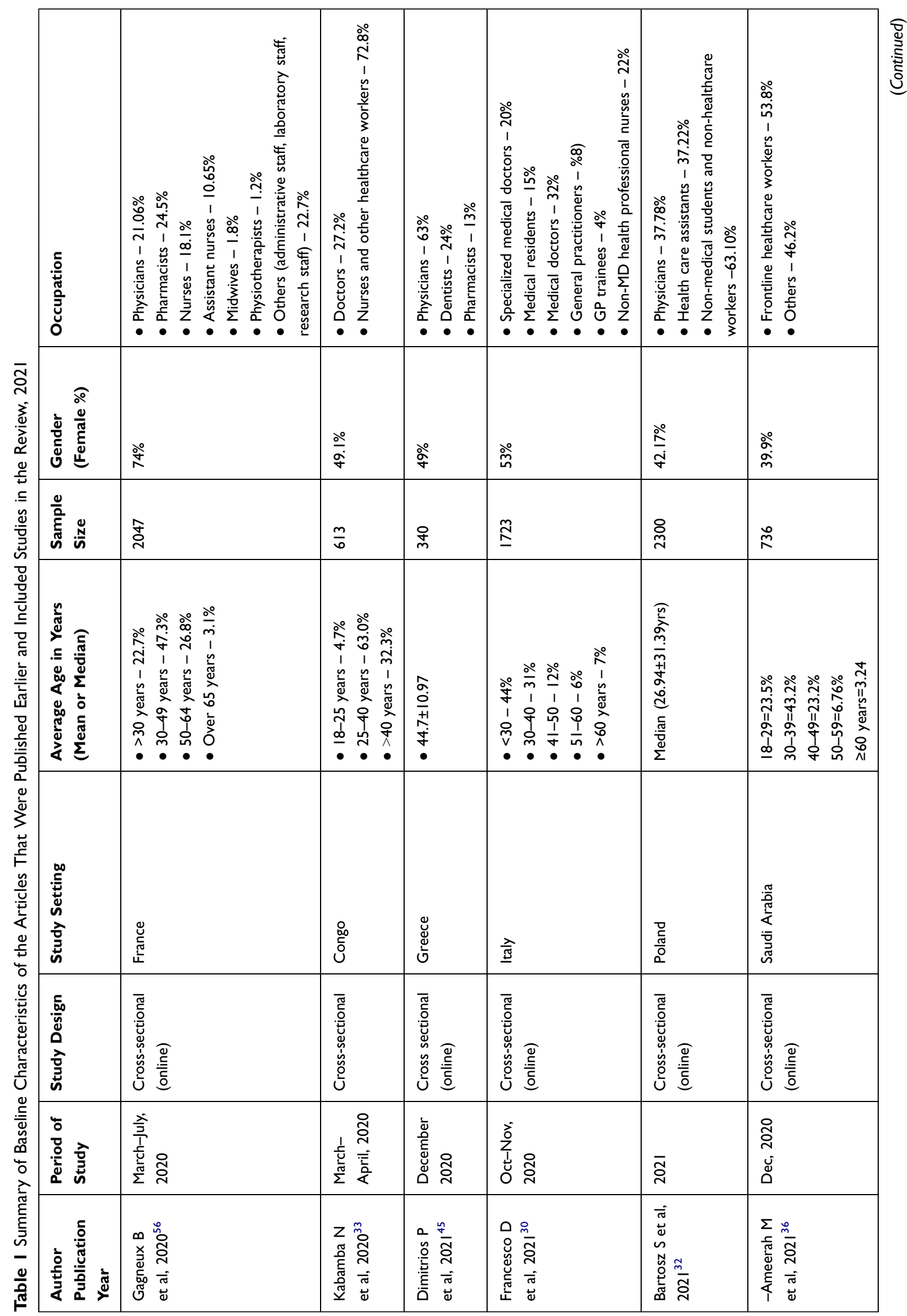




\begin{tabular}{|c|c|c|c|c|c|c|c|}
\hline 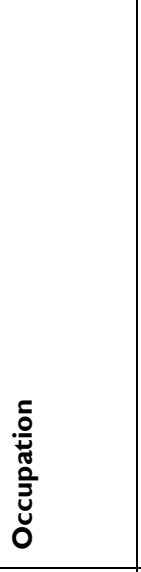 & 1 & 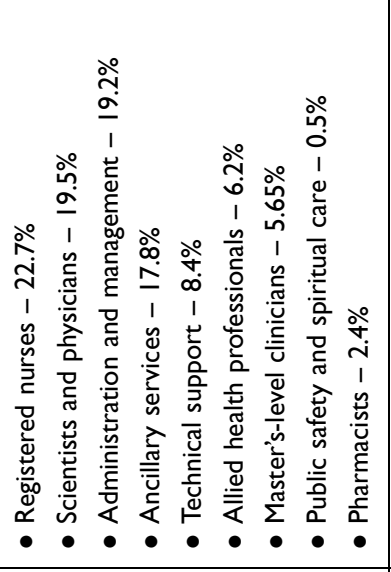 & 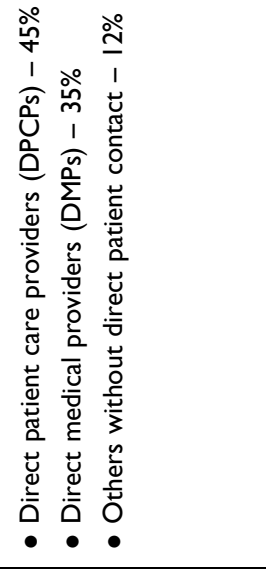 & 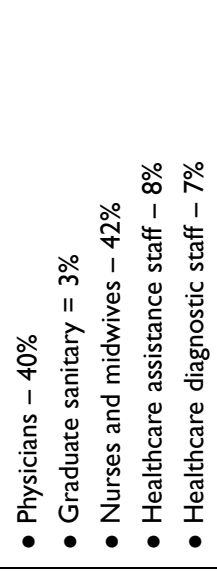 & 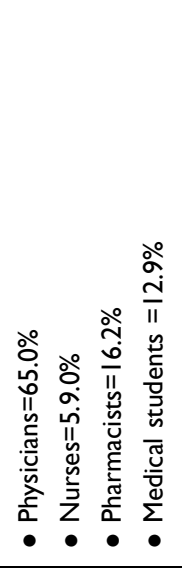 & 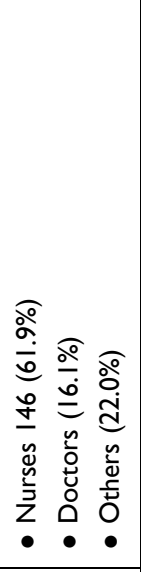 & 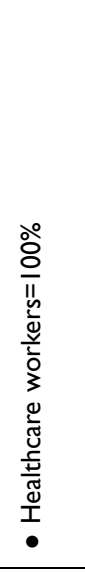 \\
\hline 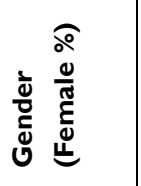 & 1 & 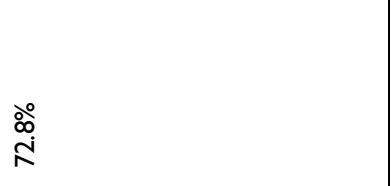 & ํํㅅ & ஸें & $\begin{array}{l}\stackrel{\circ}{\circ} \\
\text { o }\end{array}$ & $\frac{\stackrel{\circ}{\circ}}{\stackrel{x}{x}}$ & $\begin{array}{l}\stackrel{\circ}{\circ} \\
\infty \\
\infty \\
\stackrel{\infty}{n}\end{array}$ \\
\hline 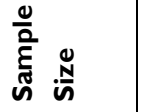 & ষ্ণ & 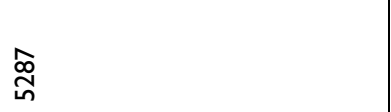 & $\begin{array}{c}\stackrel{a}{f} \\
\underset{m}{m}\end{array}$ & 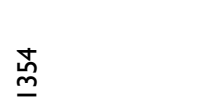 & గิ & $\stackrel{\sim}{\sim}$ & ৪ \\
\hline 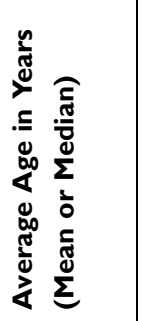 & 1 & 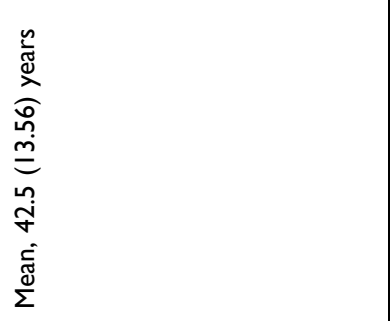 & 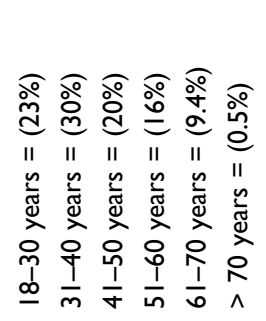 & 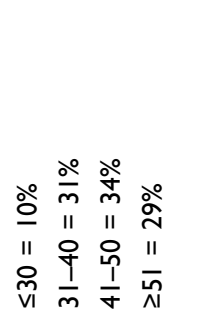 & 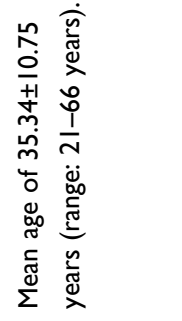 & 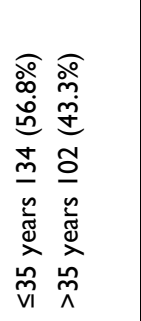 & 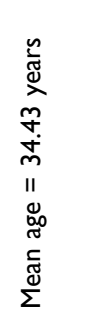 \\
\hline 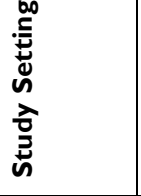 & 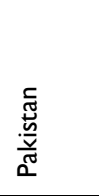 & 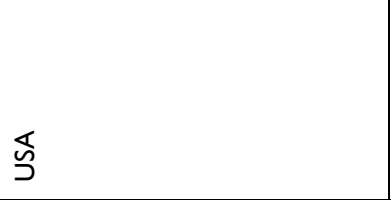 & کొ & 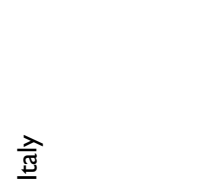 & 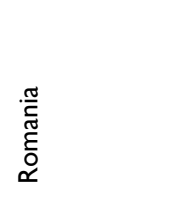 & 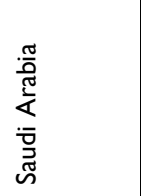 & 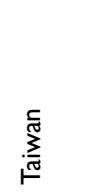 \\
\hline 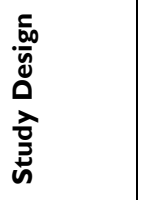 & 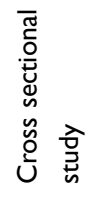 & 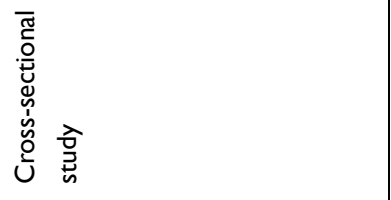 & 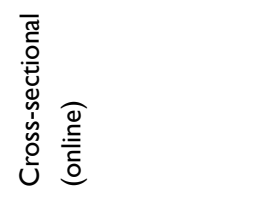 & 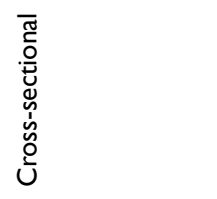 & 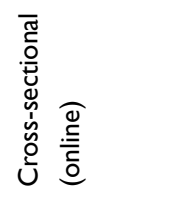 & 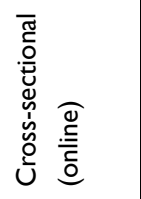 & 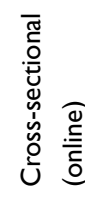 \\
\hline 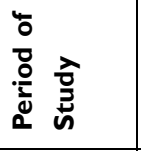 & 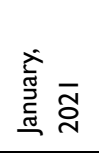 & 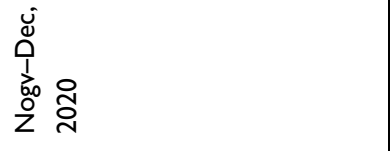 & Oั & $\overline{\widehat{d}}$ & 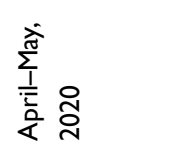 & ర్ని & 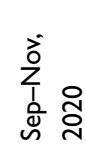 \\
\hline 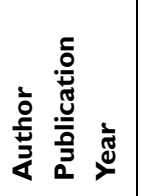 & 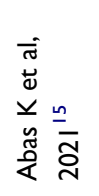 & 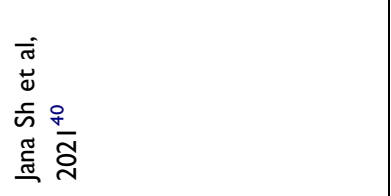 & 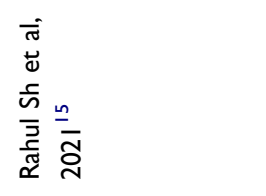 & 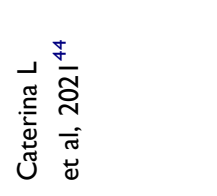 & 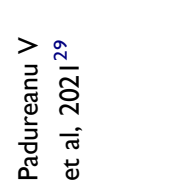 & 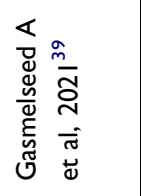 & 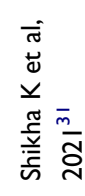 \\
\hline
\end{tabular}




\begin{tabular}{|c|c|c|c|c|c|c|c|}
\hline 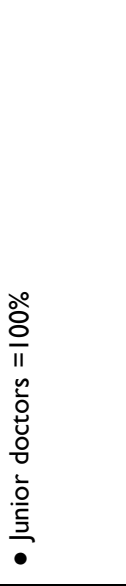 & 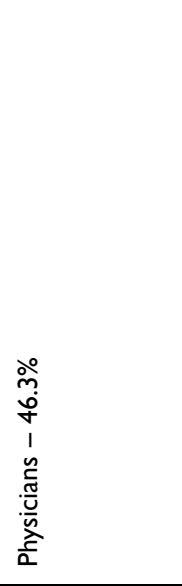 & 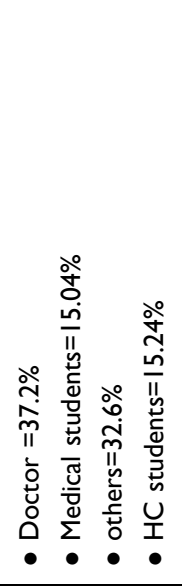 & 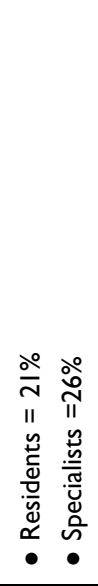 & 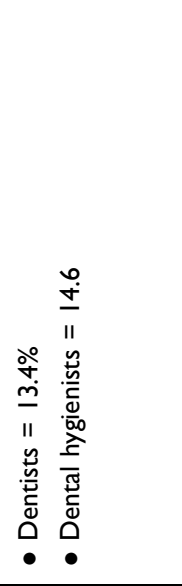 & 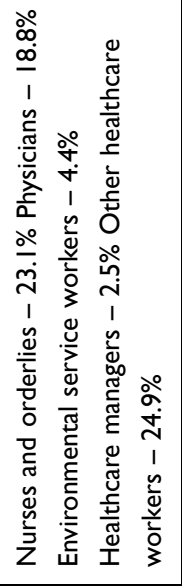 & 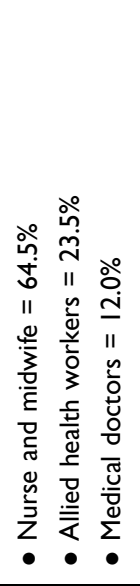 & 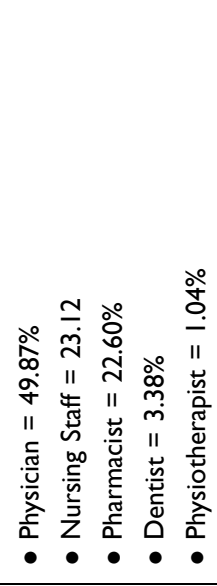 \\
\hline 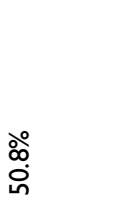 & ๖̊ㅇㅇ & ஓे̊ & ڤ̊ & 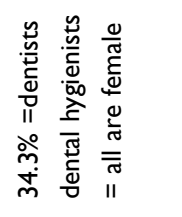 & ڤั & స్ָై & $\frac{\stackrel{\circ}{m}}{\infty}$ \\
\hline 怠 & $\stackrel{\circ}{\circ}$ & $\stackrel{\infty}{\stackrel{\infty}{N}}$ & 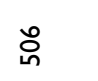 & q & $\underset{i}{\stackrel{\bar{o}}{i}}$ & $\underset{\sim}{\stackrel{్}{N}}$ & $\stackrel{\stackrel{\leftrightarrow}{\infty}}{\infty}$ \\
\hline 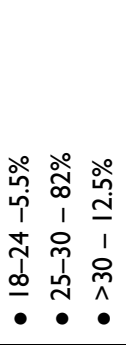 & 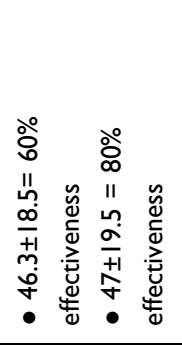 & 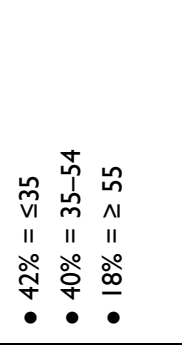 & 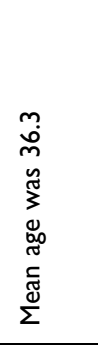 & 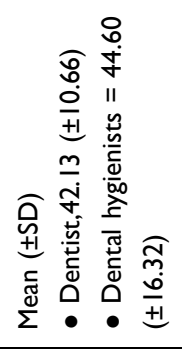 & 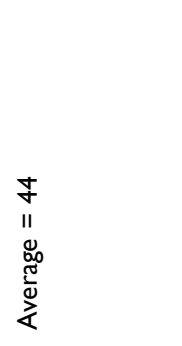 & 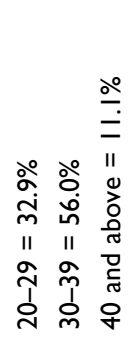 & 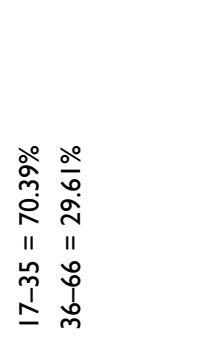 \\
\hline 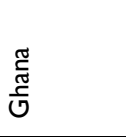 & $\begin{array}{l}\frac{\pi}{0} \\
\frac{\hat{0}}{0} \\
0\end{array}$ & $\begin{array}{l}\frac{d}{\bar{\omega}} \\
\stackrel{\partial}{0} \\
\frac{0}{\omega}\end{array}$ & 岸 & $\begin{array}{l}\overline{\bar{g}} \\
\underline{\tilde{w}} \\
\underline{\underline{u}}\end{array}$ & 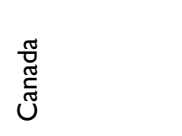 & $\begin{array}{l}\text { 苂 } \\
\text { 胥 }\end{array}$ & $\underset{\mathrm{D}}{\stackrel{\mathrm{⿱}}{\mathrm{b}}}$ \\
\hline 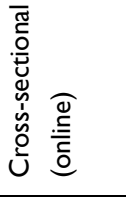 & 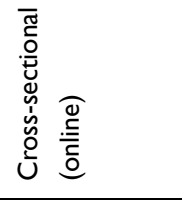 & 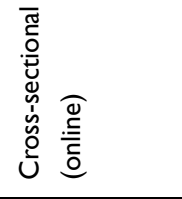 & 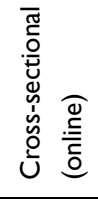 & 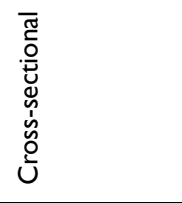 & 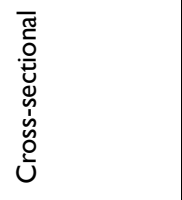 & 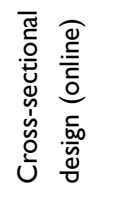 & 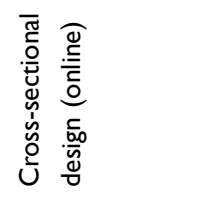 \\
\hline ָั. & $\overline{\text { ర్ }}$ & $\overline{\text { ర్ }}$ & 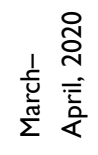 & 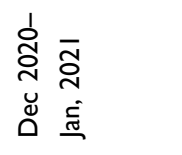 & 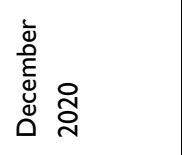 & 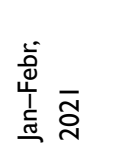 & 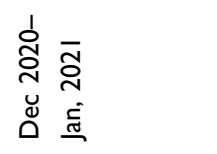 \\
\hline 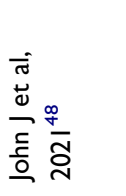 & 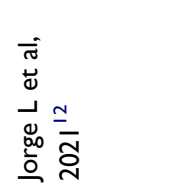 & 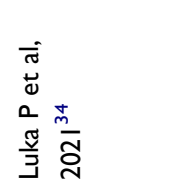 & 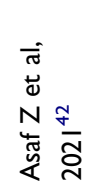 & 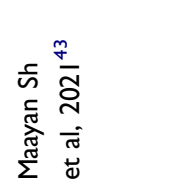 & 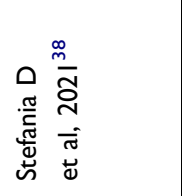 & 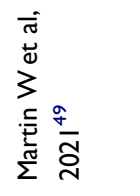 & 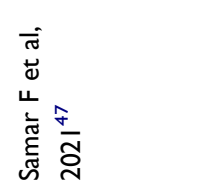 \\
\hline
\end{tabular}




\begin{tabular}{|c|c|c|c|}
\hline o & 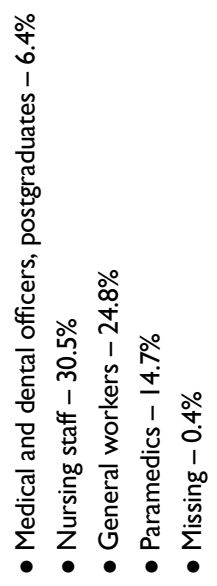 & 1 & 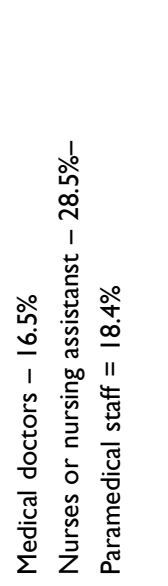 \\
\hline 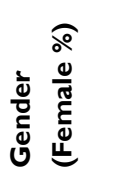 & $\frac{\stackrel{\circ}{\grave{r}}}{\wedge}$ & $\begin{array}{l}\stackrel{\circ}{+} \\
\dot{+}\end{array}$ & $\stackrel{\stackrel{\circ}{i}}{\stackrel{N}{N}}$ \\
\hline 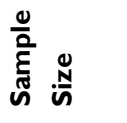 & $\stackrel{\circ}{\sim}$ & 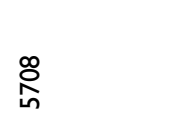 & $\underline{\underline{\Xi}}$ \\
\hline 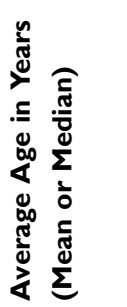 & 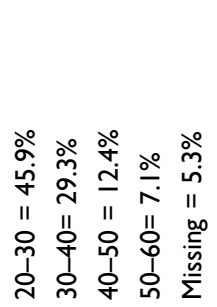 & 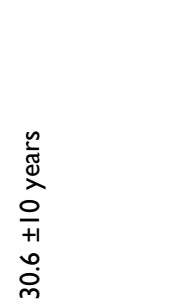 & 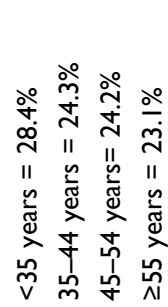 \\
\hline 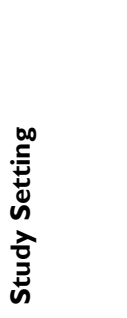 & $\begin{array}{l}\overline{\tilde{0}} \\
\text { zo }\end{array}$ & 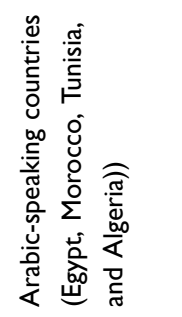 & 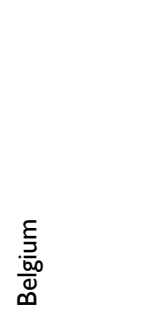 \\
\hline 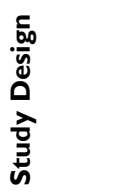 & 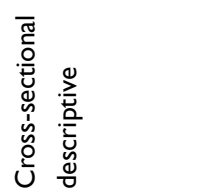 & 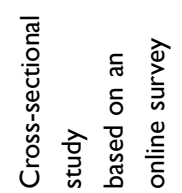 & 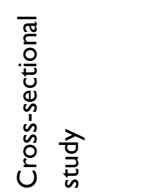 \\
\hline 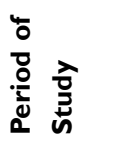 & 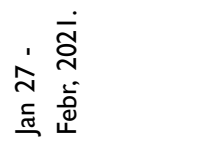 & 突 & 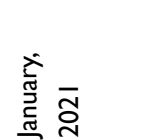 \\
\hline 尊 & 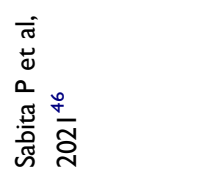 & 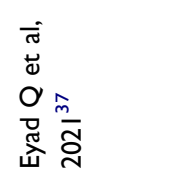 & 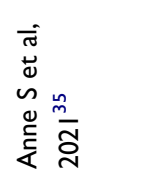 \\
\hline
\end{tabular}

Apart from the above-mentioned reports, a survey from the $\mathrm{USA}^{40}$ and Pakistan ${ }^{15}$ found that the majority of healthcare workers have to wait for about 3 months to 1 year duration and review more data to clearly state their approaches for the vaccine. In addition, considering the effectiveness of a vaccine, the Colombian ${ }^{12}$ survey showed more positive attitudes or higher percentages of healthcare workers' acceptance of the $80 \%$ vaccines effectiveness $(90.7 \%)$ compared to $60 \%$ vaccines effectiveness (77.0\%) (Table 2).

Considering professional specialty, more negative attitudes toward vaccination to COVID-19 were reported among nurses and technicians compared to other healthcare workers in a sample reported from Belgium, ${ }^{35}$ Nepal ${ }^{46}$ France, ${ }^{41}$ Slovenia, ${ }^{34}$ Israel, ${ }^{43}$ and Congo. ${ }^{33}$ In most of the studies $(n=11)$ physicians showed a more positive attitude toward vaccination against COVID-19 even though the finding remains significant only in studies from Belgium, Congo, Slovenia, and Colombia. In contrast to this, studies from the United Arab Emirates ${ }^{42}$ revealed the lowest willingness or negative attitude toward the vaccination among physicians. Furthermore, rehabilitation center workers showed a positive attitude to the COVID-19 vaccine compared to other professionals in the Canadian sample ${ }^{38}$ (Table 2).

\section{Predictors of Attitude Towards COVID- 19 Vaccination}

In the current review, different factors were observed in terms of their significance to escape from or reduce the rate of the COVID-19 vaccination approach among healthcare workers. Factors related to the attitude of healthcare workers toward COVID-19 vaccination generally include age, sex, profession, concerns about the safety of vaccines and fear of COVID-19, trust in the accuracy of the measures taken by the government in the fight against COVID19 , being vaccinated against seasonal flu during the previous season, presence of comorbid chronic illness, history of recommendation, depression symptoms in the past week, race, absence of enough clinical trials and inadequate time for the decision, education, income level, workplace, living with nuclear family, and willingness to take a rapid test.

Sex was observed to influence the attitude of healthcare workers against COVID-19 vaccination, with males demonstrating a lower anti-vaccination approach or positive attitude in the sample of studies reported from seven 
Table 2 Summary of Included Studies on the Attitude Toward COVID-19 for Vaccination and Its Determinants Among Healthcare Workers, 2021

\begin{tabular}{|c|c|c|}
\hline Author & Attitude Toward COVID-19 for Vaccination & Determinants (Significantly Associated) \\
\hline $\begin{array}{l}\text { Gagneux B } \\
\text { et al, } 2020^{56}\end{array}$ & - $76.9 \%$ would accept a COVID-19 vaccine. Of these: & $\begin{array}{l}\text { - Older age } \\
\text { - Male } \\
\text { - Fear about COVID-19 } \\
\text { - Individual perceived risk and flu vaccination during } \\
\text { previous season }\end{array}$ \\
\hline $\begin{array}{l}\text { Kabamba N } \\
\text { et al, } 2020^{33}\end{array}$ & - $27.7 \%$ = would get vaccinated if the COVID-19 vaccine was available & $\begin{array}{l}\text { - Male healthcare workers } \\
\text { - Being a doctor }\end{array}$ \\
\hline $\begin{array}{l}\text { Dimitrios P } \\
\text { et al, } 2021^{45}\end{array}$ & - High level of acceptance for COVID-19 vaccine $=78.5 \%$ & $\begin{array}{l}\text { - Age }>45 \text { years } \\
\text { - Absence of fear over vaccine safety } \\
\text { - Information received from the Greek public health } \\
\text { authorities }\end{array}$ \\
\hline $\begin{array}{l}\text { Francesco } \\
\text { D et al, } \\
2021^{30}\end{array}$ & $\begin{array}{l}\text { - } 67 \%=\text { intended to be vaccinated, } \\
\text { - } 26 \%=\text { were not sure } \\
\text { - } 7 \% \text { = declared refusal }\end{array}$ & $\begin{array}{l}\text { - Being a non-MD health professional } \\
\text { - Using Facebook as main information source about } \\
\text { antiSARS-CoV-2 vaccination }\end{array}$ \\
\hline $\begin{array}{l}\text { Bartosz S } \\
\text { et al, } 2021^{32}\end{array}$ & $\begin{array}{l}\text { - Significant level of willingness to get vaccinated as compared to the } \\
\text { control group }=82.95 \% \text { vs } 54.31 \%\end{array}$ & $\begin{array}{l}\text { - Positive history of recommended vaccinations } \\
\text { - Fear of COVID-19 of passing on the disease to } \\
\text { relatives } \\
\text { - Depression symptoms in the past week } \\
\text { - Fear of vaccination side effects }\end{array}$ \\
\hline $\begin{array}{l}\text { Ameerah M } \\
\text { et al, } 2021^{36}\end{array}$ & $\begin{array}{l}\text { - } 50.52 \% \text { of respondents were willing to have the COVID- } 19 \text { vaccine, of } \\
\text { these: } \\
\text { - Immediate acceptance }=49.7 \% \\
\text { - Delayed acceptance }=50.3 \%\end{array}$ & $\begin{array}{l}\text { \#Acceptance } \\
\text { - Being a male healthcare worker } \\
\text { - Perceiving a high risk of infection, } \\
\text { - Believing that the COVID-19 vaccine should be } \\
\text { compulsory for all citizens and } \\
\text { - residents in the country }\end{array}$ \\
\hline $\begin{array}{l}\text { Abas } \mathrm{K} \\
\text { et al, } 2021^{15}\end{array}$ & $\begin{array}{l}\text { Overall attitudes toward vaccination were positive but specific concerns } \\
\text { regarding COVID-19 vaccine are prevalent } \\
\text { - Only one-third of respondents were amenable to COVID-19 vaccination } \\
\text { immediately } \\
\text { - >I/2 of respondents preferred to defer their decision until reviewing } \\
\text { more data } \\
\text { - High percentage of respondents waiting to review more data }\end{array}$ & \\
\hline $\begin{array}{l}\text { Jana Sh } \\
\text { et al, } 2021^{40}\end{array}$ & $\begin{array}{l}\text { Overall, } 57.5 \% \text { of individuals expressed intent to receive COVID-19 } \\
\text { vaccine }\end{array}$ & $\begin{array}{l}\text { \#Acceptance } \\
\text { - Older } \\
\text { - Male } \\
\text { - White, or Asian }\end{array}$ \\
\hline $\begin{array}{l}\text { Rahul Sh } \\
\text { et al, } 2021^{15}\end{array}$ & $\begin{array}{l}56 \% \text { = majority of the HCW were not sure or would wait to review safety } \\
\text { data before getting vaccinated } \\
11 \%=\text { will like to wait for } 3 \text { months } \\
10 \%=\text { will like to wait for } 6 \text { months, and } \\
20 \%=\text { will like to wait at least I year. } \\
8 \%=\text { unwilling to take the vaccine at all }\end{array}$ & $\begin{array}{l}\text { \#Acceptance } \\
\text { - Increased with increasing age, education, and } \\
\text { income level } \\
\text { \# \#Acceptance } \\
\text { - Decreased among female, Black, Latinx and rural } \\
\text { HCWs }\end{array}$ \\
\hline
\end{tabular}

(Continued) 
Table 2 (Continued).

\begin{tabular}{|c|c|c|}
\hline Author & Attitude Toward COVID-19 for Vaccination & Determinants (Significantly Associated) \\
\hline $\begin{array}{l}\text { Caterina } L \\
\text { et al, } 2021^{44}\end{array}$ & Overall, $75 \%$ of respondents would get a COVID-19 vaccine & $\begin{array}{l}\text { \#Acceptance } \\
\text { - Younger age (<30 years) } \\
\text { - Male } \\
\text { - Patients with comorbidities }\end{array}$ \\
\hline $\begin{array}{l}\text { Padureanu } \\
\text { V et al, } \\
2021^{29}\end{array}$ & $69 \%=$ agreed with the COVID- 19 vaccine & $\begin{array}{l}\text { - Fear of getting infected } \\
\text { - Working in a designated hospital }\end{array}$ \\
\hline $\begin{array}{l}\text { Gasmelseed } \\
\text { A et al, } \\
2021^{39}\end{array}$ & $\begin{array}{l}\text { - Accept the newly developed vaccine- } 55.5 \% \\
\text { - Advocate for newly developed vaccine - } 60.1 \% \\
\text { - Both accept and advocate for newly developed vaccine - } 51.3 \%\end{array}$ & $\begin{array}{l}\text { - Female } \\
\text { - Older age } \\
\text { - Presence of chronic illnesses } \\
\text { - Having an allergy }\end{array}$ \\
\hline $\begin{array}{l}\text { Shikha K } \\
\text { et al, } 2021^{31}\end{array}$ & - Low willingness among healthcare workers - $23.4 \%$ & $\begin{array}{l}\text { - Risk perception } \\
\text { - Preventive COVID-19 infection behaviours } \\
\text { - Willingness to take rapid test }\end{array}$ \\
\hline $\begin{array}{l}\text { John J et al, } \\
2021^{48}\end{array}$ & $\begin{array}{l}\text { - Showed } 67 \% \text { of junior doctors are willing to accept the COVID } 19 \\
\text { vaccine }\end{array}$ & $\begin{array}{l}\text { FAcceptance } \\
\text { - Adverse reaction from vaccine. } \\
\text { - Female doctors } \\
\text { - Junior doctors who live with their nuclear families }\end{array}$ \\
\hline $\begin{array}{l}\text { Jorge L } \\
\text { et al, } 2021^{12}\end{array}$ & $\begin{array}{l}\text { - Between } 77.0 \% \text { and } 90.7 \% \text { of physicians accept COVID- } 19 \text { vaccination, } \\
\text { according to the scenario evaluated where the vaccine's effectiveness was } \\
60 \text { or } 80 \% \text {, respectively }\end{array}$ & $\begin{array}{l}\text { - Medical speciality } \\
\text { - Having never paid for a vaccine } \\
\text { - Recommending the administration of the vaccine to } \\
\text { their parents or people over } 70 \text { years } \\
\text { - Dispensing the vaccine to their children } \\
\text { (effectiveness of } 60 \% \text { and } 80 \% \text {. }\end{array}$ \\
\hline $\begin{array}{l}\text { Luka P et al, } \\
2021^{34}\end{array}$ & $\begin{array}{l}59 \%=\text { intended to vaccinate. Of these: } \\
\text { - } 33 \% \text { = definitively intended to participate in vaccination } \\
\text { - } 26 \% \text { = would probably agree to vaccination }\end{array}$ & $\begin{array}{l}\text { \#Acceptance } \\
\text { - Older } \\
\text { - Male } \\
\text { - Physicians } \\
\text { - Those who trust official (government) sources }\end{array}$ \\
\hline $\begin{array}{l}\text { Asaf } Z \text { et al, } \\
2021^{42}\end{array}$ & $\begin{array}{l}\text { - } 85 \%=\text { overall rate of acceptance for a COVID-19 vaccine, according to } \\
\text { our survey }\end{array}$ & \\
\hline $\begin{array}{l}\text { Maayan Sh } \\
\text { et al, } 2021^{43}\end{array}$ & $\begin{array}{l}\text { - Dental hygienists demonstrated significant negative attitudes compared } \\
\text { to dentists }\end{array}$ & $\begin{array}{l}\text { \#Refusal } \\
\text { - Unforeseen side effects Female }\end{array}$ \\
\hline $\begin{array}{l}\text { Stefania D } \\
\text { et al, } 2021^{38}\end{array}$ & - $80.9 \%$ accepted the vaccine & $\begin{array}{l}\text { \#Acceptance: Male sex, age over 50, rehabilitation } \\
\text { center workers, and occupational COVID-19 exposure } \\
\text { \#Refusal: vaccine novelty, wanting others to receive it } \\
\text { first, and insufficient time for decision-making }\end{array}$ \\
\hline $\begin{array}{l}\text { Martin W } \\
\text { et al, } 2021^{49}\end{array}$ & $\begin{array}{l}39.3 \% \text { of them indicated acceptance of COVID-19 vaccines } \\
\text { if available } \\
60.7 \% \text { indicated non-acceptance of COVID-19 vaccines }\end{array}$ & $\begin{array}{l}\text { \#Acceptance } \\
\text { - Female } \\
\text { - Relative being diagnosed with COVID-19 } \\
\text { - Trust in the accuracy of the measures taken by the } \\
\text { government in the fight against COVID-19 } \\
\text { \#Refusal } \\
\text { - Concerns about the safety of vaccines and } \\
\text { - the adverse side effects of the vaccines }\end{array}$ \\
\hline
\end{tabular}

(Continued) 
Table 2 (Continued).

\begin{tabular}{|c|c|c|}
\hline Author & Attitude Toward COVID-19 for Vaccination & Determinants (Significantly Associated) \\
\hline $\begin{array}{l}\text { Samar } F \\
\text { et al, } 2021^{47}\end{array}$ & $\begin{array}{l}21 \% \text { accepted vaccination } \\
51 \% \text { of the participants were undecided } \\
28 \% \text { refused }\end{array}$ & $\begin{array}{l}\text { \#Acceptance: } \\
\text { - Risks of COVID-19 } \\
\text { - Safety and } \\
\text { - effectiveness of the vaccine } \\
\text { \#Refusal: } \\
\text { - Absence of enough clinical trials } \\
\text { - Fear of side effects of the vaccine }\end{array}$ \\
\hline $\begin{array}{l}\text { Sabita P } \\
\text { et al, } 2021^{46}\end{array}$ & Only just over one-third (38.3\%) were willing to be vaccinated & $\neq$ Refusal $=$ vaccine safety \\
\hline $\begin{array}{l}\text { Eyad Q } \\
\text { et al, } 2021^{37}\end{array}$ & Vaccine acceptance rate in this study was $26.7 \%$ & $\begin{array}{l}\text { F Reasons for hesitancy } \\
\text { - Concerns about side effects and distrust of the } \\
\text { expedited vaccine production and healthcare policies } \\
\text { - Age } 30-59 \\
\text { - Previous or current suspected or confirmed COVID- } \\
19, \\
\text { - Female ge } \\
\text { - Not knowing the vaccine type Authorized in the } \\
\text { participant's country } \\
\text { - Not regularly receiving the influenza vaccine. }\end{array}$ \\
\hline $\begin{array}{l}\text { Anne } S \\
\text { et al, } 2021^{35}\end{array}$ & $\begin{array}{l}62.9 \%=\text { would certainly not get vaccinated } \\
37.1 \% \text { were hesitant, with different degrees of certainty }\end{array}$ & $\begin{array}{l}\neq \text { Acceptance } \\
\text { - Older } \\
\text { - Being a physician } \\
\text { - Being vaccinated against seasonal flu }\end{array}$ \\
\hline
\end{tabular}

countries $(7 / 24=29.2 \%)$ and females showing only in two studies reported from Ghana ${ }^{49}$ and Saudi Arabia. ${ }^{39}$ In contrast, more negative attitudes against COVID-19 were observed among female healthcare workers as evidenced from the surveys carried out in the USA, ${ }^{15}$ Arabic-speaking countries, ${ }^{42}$ Israel, $^{43}$ and Ghana. ${ }^{49}$

Another variable shown to affect attitude toward vaccination uptake was age. Given this, older age was found to have a significant association with a positive attitude against COVID-19 vaccination in studies reported from Belgium, ${ }^{35}$ Canada, ${ }^{38}$ France, ${ }^{41}$ Greece, ${ }^{45}$ the USA, ${ }^{15,40}$,Saudi Arabia, ${ }^{39}$ Arabic-speaking countries, ${ }^{37}$ and Slovenia. ${ }^{34}$ Conversely, a report from Italy ${ }^{44}$ exhibited associations between younger age ( $<30$ years) and positive attitude toward vaccination.

Trust of government officials or public health authorities in Ghana, ${ }^{49}$ Greece, $^{45}$ and Slovenia ${ }^{34}$ and using Facebook as the main information source in Italy $^{30}$ were also shown to have a relation with a positive attitude toward vaccination among healthcare workers. Moreover, a positive history of recommended vaccinations has also been shown to impact the uptake of the vaccine positively among healthcare workers in Poland ${ }^{32}$ and Colombia. ${ }^{12}$
Furthermore, psychological factors such as fear about COVID-19, fear of passing on the disease to relatives, presence of depression symptoms in the past week, perceiving a high risk of infection, absence of fear over vaccine safety ${ }^{33,46}$ and compulsory pressure from the government to get the vaccine for all citizens positively impacted the uptake of the vaccine among healthcare workers. $^{29,32,36,41}$ However a survey from Taiwan ${ }^{31}$ reported risk perception of illness has a positive association with COVID-19 vaccination.

Other factors associated with a positive attitude toward vaccine or a lower anti-vaccination approach includes having never paid for a vaccine, dispensing the vaccine to their children (particularly those of effectiveness of $60 \%$ and $80 \%),{ }^{12}$ working in a designated hospital, ${ }^{29}$ higher level of educational status, and income level, ${ }^{15}$ being a non-MD health professional ${ }^{30}$ and being junior doctors who live with their nuclear families. ${ }^{48}$ Also, clinical factors such as the presence of chronic illnesses, flu vaccination during the previous season, and having allergies ${ }^{35,39,44}$ were reported to have a positive association with uptake of the COVID-19 vaccine. 
Considering contributing factors for the negative attitudes or higher anti-vaccination approach among healthcare workers, the following factors were identified in prior studies: side effects of vaccine, ${ }^{32,37,43,47-49}$ vaccine novelty, wanting others to receive it first, insufficient time for decisionmaking, ${ }^{37,38,46,49}$ Black, Latin and rural healthcare workers, relative being diagnosed with COVID-19, ${ }^{49}$ absence of enough clinical trials, ${ }^{47}$ not regularly receiving the influenza vaccine, not knowing the vaccine type authorized in the participant's country, previous or current suspected or confirmed COVID-19, ${ }^{37}$ risks of COVID-19, safety and effectiveness of the vaccine, ${ }^{47}$ preventive COVID-19 infection behaviours, and willingness to take rapid test. In contrast to this report, the report from the USA ${ }^{40}$ showed that Whites and Asians were more likely to get vaccinated for COVID-19 than other groups.

\section{Discussion}

Although the World Health Organization and its respective partners are working tirelessly to distribute the COVID-19 vaccine, they have been facing challenges in some countries to administer it appropriately. In this review, we have critically reviewed the published papers that showed the attitude of healthcare workers toward the COVID-19 vaccine, which is among the major challenges the world is currently facing. The review revealed that, in about twothirds of the studies, respondents showed a positive attitude ( $\geq 50 \%)$ toward COVID-19 vaccination. Nonetheless, negative attitude $(<50 \%)$ against vaccination was reported in about one-quarter of the studies. A lower and higher rate of acceptance or anti-vaccination approach toward COVID-19 was reported in Egypt and the United Arab Emirates, respectively. The review indicated more negative attitudes were observed in a survey among African and Asian studies, which calls for designing an intervention to enhance the willingness of healthcare providers in these settings. In other respects, previous studies reported among public health professionals indicate that more than half $(57.1 \%)$ are aware of all vaccines generally recommended for health workers. It also shows that public health professionals who have been vaccinated are more likely to advocate vaccination for uncertain people and patients. ${ }^{50}$

The review results showed that older healthcare workers were found to have a good attitude toward the COVID19 vaccine. This may be concerning the fact that the impact of the pandemic is more severe with increasing age, and older unvaccinated people are more likely to be hospitalized or die from COVID-19. ${ }^{51}$ Studies showed that more than $80 \%$ of COVID-19 deaths occur in people over age 65 , and more than $95 \%$ of COVID-19 deaths occur in people older than $45 .^{51}$ In support of this finding, with advanced age muscles become weaker and the anatomy of lungs changes, which causes older people to become less efficient in moving air and mucus out of the body resulting in a lowering of the defense system that the lungs and immune systems can provide. ${ }^{52}$ Being aware of the fact that, when infected, older people are more likely to get severely ill from COVID-19 could motivate or enhance their participation in every measure to tackle the pandemic.

Female healthcare workers were found to be less willing to accept the COVID-19 vaccine as compared to males. The reason may be due to the relatively good health-seeking behaviour of males and their valuing of advice about the effectiveness of COVID-19 vaccines as supported and evidenced by different behavioural approach theories, including the theory of planned behaviour and the theory of reasoned action. ${ }^{53-56}$ Additionally, being a medical professional (medical doctors, physicians, specialists) was related to a positive attitude toward the COVID-19 vaccination. The evidence was conclusive as a positive attitude was associated with a higher level of education. $^{15,53,57}$

As a result of the safety of COVID-19 vaccines, healthcare workers may have a negative attitude toward the vaccine. Some people notice side effects of the COVID19 vaccine such as pain or swelling, blood clotting, and headache. ${ }^{58}$ Healthcare workers with co-morbid chronic illnesses were found to have a positive attitude toward COVID-19 vaccines. This may be related to the high risk of COVID-19 related lethality when combined with the presence of chronic medical illness. ${ }^{59}$

In agreement with a previous research finding, ${ }^{56}$ healthcare workers who had taken influenza vaccine during previous seasons had a good attitude toward the COVID-19 vaccine. This could be related to the role of false information and is thought to be a barrier to combat COVID-19 in the current review and previous report. ${ }^{60} \mathrm{As}$ the healthcare workers' explanations or suggestions have a positive influence on attitude toward the vaccine, ${ }^{56,61}$ trustworthiness in the accuracy of measures taken, healthcare policies, and recommendations by the government also had a similar influence on the vaccine uptake.

The COVID-19 outbreak poses a significant challenge for all countries, creating an unprecedented need for international solidarity and a coordinated global response. So, 
in addition to the attitude of professionals toward the vaccine, global funds for low-income countries need to be maintained.

\section{Limitations}

This work was subjected to several limitations. First, an obvious limitation of the review was the cross-sectional nature of the included studies, with the majority being reported online (possible sampling bias). Again in the review, we have not used other databases such as CINAHL, EMBASE, PsycInfo, and COCHRANE.

It is familiar that change in behaviour of the individuals is inevitable So the finding of this study may be subject to unexplained factors that impact the behaviours of the respondents, which include unpredictable variants of COVID infection, doses, and so on. So prospective reviews could target similar respondents to identify potential predictors for attitude variation in line with trends of the time. Furthermore, assessment of the quality of included articles was rated based on the interest of the authors using a quality assessment tool for the observational cohort and cross-sectional studies. Finally, the result of synthesis or meta-analysis was not investigated; rather, we conducted a qualitative review to make a more comprehensive understanding.

\section{Conclusion}

Although most studies report that healthcare workers have a positive attitude toward COVID-19 vaccination, quite a few surveys mention negative attitudes towards the use of vaccines, which may reflect missed opportunities or challenges for the international efforts aimed at mitigating the pandemic. Because this can be challenging, it is necessary to maintain communication between different levels of health authorities, public health campaigns, and recommendations from public health professionals to build trust and accountability. There is still a need to continue to make more effort to change the attitudes of the uncertain population, to increase the uptake of the vaccine, and to deal with the multi-faceted impact of infection.

\section{Abbreviations}

COVID, Coronavirus disease; NIH, National Institute of Health; PRISMA, Preferred Reporting Items for Systematic Reviews and Meta-analyses; PROSPERO, Prospective Register of Ongoing Systematic Review.

\section{Data Sharing Statement}

The datasets used are available from the corresponding author on reasonable request.

\section{Funding}

No funding was received.

\section{Disclosure}

The authors report no conflicts of interest in this work.

\section{References}

1. WHO "WHO coronavirus disease (COVID-19) Dashboard," Weekly operational update on COVID-19, July 05, 2021. Available from: https://www.who.int/docs/default-source/coronaviruse/weeklyupdates/wou_2021_5-july_cleared-.pdf?sfvrsn=e69c6bdf_3\&down load $=$ true.

2. Carla K, Johnson A. Nearly all COVID deaths in U.S. Are Now Among Unvaccinated. Health Sci. 2020;21:1023-1034.

3. Carla K. Nearly all COVID deaths in U.S. Are now among unvaccinated; 2021.

4. Mbaeyi S. Use of Pfizer-BioNTech COVID-19 vaccine: clinical considerations. CDC; 2020.

5. Larson HJ, Smith DMD, Paterson P, et al. Measuring vaccine confi dence: analysis of data obtained by a media surveillance system used to analyse public concerns about vaccines. Lancet Infect Dis. 2013;13 (7):606-613. doi:10.1016/S1473-3099(13)70108-7

6. Nzaji MK, Ngombe LK, Mwamba GN, Miema JM, Lungoyo CL, Mwimba BL. Acceptability of vaccination against COVID-19 among healthcare workers in the democratic Republic of the Congo. Pragmatic Obs Res. 2020;11:103-109. doi:10.2147/POR.S271096

7. On K, Li K, In W, Tang A. Influenza vaccine uptake, COVID-19 vaccination intention and vaccine hesitancy among nurses: a survey. Int J Nurs Stud. 2020;114(1):103854.

8. Akarsu B, Canbay D, Duygu Ö, Baser A, Fidancı İ, Cankurtaran M. While studies on COVID-19 vaccine is ongoing, the public' $\mathrm{s}$ thoughts and attitudes to the future COVID-19 vaccine. Infect Dis. 2020;75(11):1-10.

9. Szmyd B, Bartoszek A, Karuga FF, Staniecka K, Błaszczyk M, Radek M. Medical students and SARS-CoV-2 vaccination: attitude and behaviors. Vaccines. 2021;9(2):1-12. doi:10.3390/vaccines9020 128

10. James PB, Rehman IU, Bah AJ, Lahai M, Cole CP. An assessment of healthcare professionals ' knowledge about and attitude towards influenza vaccination in Freetown Sierra Leone: a cross-sectional study. BMC Public Health. 2017;17(692):1-8. doi:10.1186/s12889017-4700-2

11. Paris C, Bénézit F, Geslin M. Since January 2020 Elsevier has created a COVID-19 resource centre with free information in English and Mandarin on the novel coronavirus COVID- 19. The COVID-19 resource centre is hosted on Elsevier Connect, the company' s public news and information. Infect Dis Now. 2021;51 (5):484-487. doi:10.1016/j.idnow.2021.04.001

12. Alvarado-socarras JL, Vesga-varela AL, Quintero-lesmes DC, et al. Perception of covid-19 vaccination amongst physicians in Colombia. Vaccines. 2021;9(3):1-15. doi:10.3390/vaccines9030287

13. Fisher KA, Bloomstone SJ, Walder J, Crawford S, Fouayzi H, Mazor KM. Attitudes toward a potential SARS-CoV-2 vaccine: a survey of US adults. Ann Intern Med. 2020;173(12):964-973. doi:10.7326/ M20-3569 
14. Grech V, Gauci C, Agius S. Vaccine hesitancy among Maltese healthcare workers toward influenza and novel COVID-19 vaccination. Early Hum Dev J. 2020. doi:10.1016/j.earlhumdev.2020.105213

15. Shekhar R, Sheikh AB, Upadhyay S, et al. COVID-19 vaccine acceptance among health care workers in the United States. Vaccines. 2021;9(2):1-18. doi:10.3390/vaccines9020119

16. Brand SR, Degenhardt TP, Person K, et al. A Phase 2, Randomized, double-blind, placebo-controlled, dose-ranging study to evaluate the efficacy and safety of orally-administered VT-1161 in the treatment of recurrent. Am J Obstet Gynecol. 2018;218(6):624.e1-624.e9. doi:10.1016/j.ajog.2018.03.001

17. Phadke VK, Bednarczyk RA. Association between vaccine refusal and vaccine-preventable diseases in the United States: a review of measles and pertussis. Clin Rev. 2016;315(11):1149-1158.

18. Detoc M, Bruel S, Frappe P, Tardy B, Botelho-nevers E, Gagneuxbrunon A. Intention to participate in a COVID-19 vaccine clinical trial and to get vaccinated against COVID-19 in France during the pandemic. Vaccine. 2020;38(45):7002-7006. doi:10.1016/j.vaccine.2020.09.041

19. Reiter PL, Pennell ML, Katz ML. Acceptability of a COVID-19 vaccine among adults in the United States: how many people would get vaccinated? Vaccine. 2021;38(42):6500-6507. doi:10.1016/j. vaccine.2020.08.043

20. Wheeler M, Buttenheim AM, Wheeler M, Buttenheim AM. Parental vaccine concerns, information source, and choice of alternative immunization schedules. Hum Vaccin Immunother. 2013;9(8):5515.

21. Patricia M, Stokley S, Halsey NA. Factors associated with refusal of childhood vaccines among parents of school-aged children. Arch Pediatr Adolesc Med. 2005;159(5):470-476. doi:10.1001/archpedi. 159.5.470

22. Omer SB, Salmon DA, Orenstein WA, Halsey N. Vaccine refusal, mandatory immunization, and the risks of vaccine-preventable diseases. New Engl J Med Spec. 2009;360(19):1981-1988. doi:10.1056/ NEJMsa0806477

23. Gallè F, Sabella EA, Roma $P$, et al. Knowledge and acceptance of COVID-19 vaccination among undergraduate students from central and southern Italy. Vaccines. 2021;9(6):1-13. doi:10.3390/vaccines9060638

24. Anwar A, Malik M, Raees V, Anwar A. Role of mass media and public health communications in the COVID-19 pandemic. Cureus. 2020;12:9.

25. Williams L, Gallant AJ, Rasmussen S, et al. Towards intervention development to increase the uptake of COVID-19 vaccination among those at high risk: outlining evidence-based and theoretically informed future intervention content. Br J Health Psychol. 2020;25 (4):1039-1054. doi:10.1111/bjhp.12468

26. Lemaitre M, Meret T, Rothan-tondeur M, Belmin J. Effect of influenza vaccination of nursing home staff. Am Geriatr Soc. 2009;57 (9):1580-1586. doi:10.1111/j.1532-5415.2009.02402.x

27. Moher D, Liberati A, Tetzlaff J, et al. Preferred reporting items for systematic reviews and meta-analyses: the PRISMA statement. PLoS Med. 2009;6(7):7. doi:10.1371/journal.pmed.1000097

28. Studies. NI of H (2014). QAT for OC and C-S. Quality assessment tool for observational cohort and cross-sectional studies. Bethesda, MD: National Institutes of Health, Department of Health and Human Services; 2014.

29. Craiova P. Perceptions of Covid-19 vaccination among healthcare perceptions of Covid-19 vaccination among healthcare. Prev Med Lab. 2020;124(10):3.

30. Di Gennaro F, Murri R, Segala FV, et al. Attitudes towards antiSARS-CoV2 vaccination among healthcare workers: results from a national survey in Italy. Viruses. 2021;13(3):1-11. doi:10.3390/ v13030371

31. Kukreti S, Lu MY, Lin YH, et al. Willingness of taiwan's healthcare workers and outpatients to vaccinate against covid-19 during a period without community outbreaks. Vaccines. 2021;9(3):1-10. doi:10. 3390/vaccines 9030246
32. Szmyd B, Karuga FF, Bartoszek A, et al. Attitude and behaviors towards sars-cov-2 vaccination among healthcare workers: a crosssectional study from Poland. Vaccines. 2021;9(3):1-14.

33. Kabamba nzaji M, Kabamba ngombe L, Ngoie mwamba G, et al. Acceptability of vaccination against covid-19 among healthcare workers in the democratic Republic of the Congo. Pragmatic Obs Res. 2020;11:103-109.

34. Petravić L, Arh R, Gabrovec T, et al. Factors affecting attitudes towards covid-19 vaccination: an online survey in Slovenia. Vaccines. 2021;9(3):1-15. doi:10.3390/vaccines9030247

35. Spinewine A, Pétein C, Evrard P, et al. Attitudes towards covid-19 vaccination among hospital staff-understanding what matters to hesitant people. Vaccines. 2021;9(5):1-13. doi:10.3390/vaccines 9050469

36. Qattan AMN, Alshareef N, Alsharqi O, Al Rahahleh N, Chirwa GC, Al-Hanawi MK. Acceptability of a COVID-19 vaccine among healthcare workers in the Kingdom of Saudi Arabia. Front Med. 2021;8(3):1-12. doi:10.3389/fmed.2021.644300

37. Qunaibi E, Basheti I, Soudy M, Sultan I. Hesitancy of Arab healthcare workers towards covid-19 vaccination: a large-scale multinational study. Vaccines. 2021;9(5):1-13. doi:10.3390/vaccines9050446

38. Dzieciolowska S, Hamel D, Gadio S, et al. Covid-19 vaccine acceptance, hesitancy, and refusal among Canadian healthcare workers: a multicenter survey. Am J Infect Control. 2021;000:1-6.

39. Ahmed G, Almoosa Z, Mohamed D, et al. Healthcare provider attitudes toward the newly developed COVID-19 vaccine: cross-sectional study. Nurs Rep. 2021;11(1):187-194. doi:10.3390/nursrep11010018

40. Shaw J, Stewart T, Anderson KB, et al. Assessment of U.S. health care personnel (HCP) attitudes towards COVID-19 vaccination in a large university health care system. Clin Infect Dis. 2021;2019. doi:10.1093/cid/ciab054

41. Salazar M, Barochiner J. Intention to get vaccinations against COVID-19 in French healthcare workers during the first pandemic wave: a cross-sectional survey. Ann Oncol. 2020;108(1):168-173.

42. Zigron A, Dror AA, Morozov NG, et al. COVID-19 vaccine acceptance among dental professionals based on employment status during the pandemic. Front Med. 2021;8(2):1-5. doi:10.3389/fmed.2021.618403

43. Shacham M, Greenblatt-Kimron L, Hamama-Raz Y, et al. Increased covid-19 vaccination hesitancy and health awareness amid covid-19 vaccinations programs in Israel. Int J Environ Res Public Health. 2021;18(7):3804. doi:10.3390/ijerph18073804

44. Ledda C, Costantino C, Cuccia M, Maltezou HC, Rapisarda V. Attitudes of healthcare personnel towards vaccinations before and during the covid-19 pandemic. Int $J$ Environ Res Public Health. 2021;18(5):1-11. doi:10.3390/ijerph18052703

45. Papagiannis D, Rachiotis G, Malli F, et al. Acceptability of covid-19 vaccination among Greek health professionals. Vaccines. 2021;9 (3):1-7. doi:10.3390/vaccines 9030200

46. Paudel S, Palaian S, Shankar PR, Subedi N. Risk perception and hesitancy toward COVID-19 vaccination among healthcare workers and staff at a medical college in Nepal. Risk Manag Healthc Policy. 2021;14:2253-2261. doi:10.2147/RMHP.S310289

47. Fares S, Elmnyer MM, Mohamed SS, Elsayed R. COVID-19 vaccination perception and attitude among healthcare workers in Egypt. $J$ Prim Care Community Health. 2021;12:21501327211013303

48. Annan JJK, Norman BR, Mensah B, Enimil A, Kokuro C. Willingness to accept vaccination against SARS-cov-2: a survey of junior doctors. World J Adv Res Rev. 2021;9(3):159-166. doi:10. 30574/wjarr.2021.9.3.0092

49. Agyekum MW, Afrifa-Anane GF, Kyei-Arthur F, Addo B. Acceptability of COVID-19 vaccination among health care workers in Ghana. $A d v$ Public Heal. 2021;2021. doi:10.1101/ 2021.03.11.21253374

50. Montagna MT, De Giglio O, Napoli C, et al. Adherence to vaccination policy among public health professionals: results of a national survey in Italy. Vaccines. 2020;8(3):1-16. doi:10.3390/vaccines 8030379 
51. Prevention. $\mathrm{C}$ for DC and. Centers for disease control and prevention [Internet]; 2021. Available from: https://search.cdc.gov/search/index. html\#advanced. Accessed September 3, 2021.

52. Umanskaya A. Study explains why muscles weaken with age and points to possible therapy; 2011.

53. Rapisarda V, Vella F, Ledda C, Barattucci M, Ramaci T. What Prompts doctors to recommend COVID-19 vaccines: is it a question of positive emotion? Vaccines. 2021;9(6):578. doi:10.3390/vaccines 9060578

54. Buess M. Metastasiertes nierenzellkarzinom: heutige therapeutische optionen. Tagliche Prax. 2012;53(1):51-58.

55. Hill RJ, Fishbein M, Ajzen I. Belief, Attitude, Intention and Behavior: an Introduction to Theory and Research. Contemp Sociol. 1977;6(2):244-245. doi:10.2307/2065853

56. Gagneux-Brunon A, Detoc M, Bruel S, et al. Intention to get vaccinations against COVID-19 in French healthcare workers during the first pandemic wave: a cross-sectional survey. J Hosp Infect. 2021;108:168-173. doi:10.1016/j.jhin.2020.11.020

57. Rhodes A, Hoq M, Measey M-A. Intention to vaccinate against COVID-19 in Australia. Lancet. 2020;21(5):2020-2021.
58. Johns Hopkins Medicine. Conditions and Diseases | Johns Hopkins Medicine [Internet]. John Hopkin; 2020. Available from: https:// www.hopkinsmedicine.org/health/conditions-and-diseases. Accessed September 3, 2021.

59. Guan W, Ni Z, Hu Y, et al. Clinical characteristics of coronavirus disease 2019 in China. N Engl J Med. 2020;382(18):1708-1720. doi:10.1056/NEJMoa2002032

60. Nasir NM, Baequni B, Nurmansyah MI. Misinformation related to Covid-19 in Indonesia. $J$ Adm Kesehat Indones. 2020;8(2):51.

61. Dini G, Toletone A, Sticchi L, Orsi A, Bragazzi NL, Durando P. Influenza vaccination in healthcare workers: a comprehensive critical appraisal of the literature. Hum Vaccines Immunother. 2018;14 (3):772-789. doi:10.1080/21645515.2017.1348442

62. Page MJ, McKenzie JE, Bossuyt PM, Boutron I, Hoffmann TC, Mulrow CD, et al. The PRISMA 2020 statement: an updated guideline for reporting systematic reviews. BMJ. 2021;372:n71. doi:10.1136/bmj.n71.
Infection and Drug Resistance

\section{Publish your work in this journal}

Infection and Drug Resistance is an international, peer-reviewed openaccess journal that focuses on the optimal treatment of infection (bacterial, fungal and viral) and the development and institution of preventive strategies to minimize the development and spread of resistance. The journal is specifically concerned with the epidemiology of
Dovepress

antibiotic resistance and the mechanisms of resistance development and diffusion in both hospitals and the community. The manuscript management system is completely online and includes a very quick and fair peerreview system, which is all easy to use. Visit http://www.dovepress.com/ testimonials.php to read real quotes from published authors. 